\title{
Entorno familiar del adulto mayor de los centros vida de la ciudad de Villavicencio, Colombia*
}

\author{
Family Environment of the Elderly in the Centros Vida in the City of Villavicencio, Colombia \\ Contexto familiar do idoso nos Centros Vida da cidade de Villavicencio, Colômbia
}

\author{
Emilce Salamanca-Ramos ${ }^{\text {a }}$ \\ Universidad de los Llanos, Colombia \\ esalamanca@unillanos.edu.co \\ ORCID: http://orcid.org/000-0002-3549-7067 \\ Zulma Johana Velasco Páez \\ Universidad de los Llanos, Colombia \\ ORCID: http://orcid.org/000-0003-1728-5631 \\ Clara Delcy Díaz Viatela \\ Universidad de los Llanos, Colombia \\ ORCID: http://orcid.org/0000-0002-5576-7351
}

DOI: https://doi.org/0.11144/Javeriana.ie21-1.efam Redalyc: http://www.redalyc.org/articulo.oa? id $=145257605005$

Fecha de recepción: 09 Octubre 2017

Fecha de aprobación: 04 Julio 2018

\section{Resumen:}

Introducción: En la actualidad, el envejecimiento de la población despierta interés, debido al aumento de la esperanza de vida. Objetivo: Conocer el entorno familiar de los adultos mayores de los Centros Vida (CV) del municipio de Villavicencio, con el propósito de construir, a partir de los resultados, elementos orientadores para la formación de cuidadores profesionales y el mejoramiento de la calidad de vida del adulto mayor. Método: Perspectiva cualitativa con enfoque etnográfico. Se incluyeron tres CV seleccionados al azar; de cada CV participó un cuidador y un adulto mayor con su familia. Se utilizó observación no participante y entrevista abierta. En total, se realizaron nueve entrevistas que fueron transcritas y convertidas en unidades textuales, con el apoyo del software $\mathrm{N}$-vivo 11. El análisis fue simultáneo con el trabajo de campo. Se realizó codificación y categorización. Resultados: Se identificaron tres categorías: 1) entre la aceptación o la resignación de ser un adulto mayor, 2) los menesteres de la vejez y 3 ) las relaciones de la familia cuando se tiene un adulto mayor. Conclusión: Para los adultos mayores el envejecimiento es una etapa de pérdida, abandono y sufrimiento. Para la familia, es una etapa muy difícil cuando no se tiene condiciones socioeconómicas suficientes, se requiere apoyo y aceptación de los cambios producidos en el adulto mayor. Para los cuidadores de los $\mathrm{CV}$ es una etapa que requiere mucho apoyo de la familia y la sociedad. Palabras clave: adulto mayor, familia, relaciones familiares, envejecimiento.

\section{Abstract:}

Introduction: The aging of people is currently awakening interest due to the increase in the life expectancy. Objective: To know the family environment of the elderly in the Centros Vida [Life Centers] in the city of Villavicencio and use the results to build guiding elements intended to educate professional caregivers, thus improving the elderly's quality of life. Method: This work used a qualitative perspective with an ethnographic approach. Three CVs were selected randomly. From each CV a caregiver, an elderly person and his/her family participated in the study. Non-invasive observations and open interviews were used in the study. Nine interviews were completed, then typed and converted in textual units by using the software $\mathrm{N}$-vivo 11 . The analysis was carried out simultaneously with the field work. The data were then subject to coding and categorization. Results: Three categories were identified: (1) either accepting or resigning oneself to be an elderly person; (2) the needs of the old age, and (3) the family 
relationships when there is an elderly person in it. Conclusion: For the elderly, aging is a stage of life coming with losses, neglect, and suffering. This is a very difficult stage when the family does not have enough socio-economic resources; the family needs to accept and support the changes undergone by the elderly. To the caregivers working in the CVs, this is a stage of life requiring a lot of support by the family and the society.

Keywords: elderly, family, family relationships, aging.

\section{Resumo:}

Introdução: No momento atual o envelhecimento da população desperta interesse devido ao acréscimo da esperança de vida. Objetivo: Conhecer o contexto familiar dos idosos nos Centros Vida (CV) do município de Villavicencio, objetivando construir, a partir dos resultados, elementos orientadores para a formação de cuidadores professionais e o melhoramento da qualidade de vida do idoso. Método: Perspectiva qualitativa com abordagem etnográfica. Incluíram-se três CV selecionados aleatoriamente; por cada CV participou um cuidador e um idoso com a família. Observação não participante e entrevista aberta foram feitas. Em total, nove entrevistas foram realizadas, sendo transcritas e convertidas em unidades textuais, com suporte do software N-vivo 11. A análise foi simultânea com o trabalho de campo. Realizou-se codificação e categorização. Resultados: três categorias foram identificadas: 1) entre aceitação ou resignação de ser idoso, 2) os afazeres da velhice e 3) os relacionamentos da família quando tem um idoso em casa. Conclusão: Para os idosos o envelhecimento é um estágio de perda, abandono e sofrimento. Para a família, é um estágio muito difícil quando não houver condições socioeconômicas suficientes, é preciso apoio e aceite das mudanças produzidas no idoso. Para os cuidadores dos CV é um estágio que requer muito suporte da família e da sociedade. Palavras-chave: idoso, família, relacionamentos familiares, envelhecimento.

\section{Introducción}

Según lo descrito por la Organización Mundial de la Salud (OMS) en 2015, el envejecimiento de la población puede considerarse un éxito de las políticas de salud pública y el desarrollo socioeconómico; pero también constituye un reto para la sociedad. Actualmente, Colombia se clasifica en un estadio avanzado de transición demográfica y en una etapa moderada de envejecimiento, con un $10 \%$ de su población en el grupo de edad de 60 años o más (1), lo que lleva a cambios estructurales fundamentales, como la composición de las familias, las migraciones, los procesos de urbanización y los cambios de roles al mercado laboral.

De acuerdo con la OMS, el envejecimiento implica cambios en los patrones de enfermedad, como son las enfermedades infecciosas (responsables de altas tasas de mortalidad) y las crónicas (generadoras de discapacidad y deterioro en la calidad de vida); a la vez que influye en las condiciones económicas y financieras de los países. La situación para los adultos mayores es preocupante cuando hay dificultades económicas en la familia. En los tiempos actuales, la situación de vejez transmite la sensación que el envejecimiento de la población constituye una amenaza potencialmente inmanejable que afecta el bienestar general, de los hogares y de las sociedades.

De acuerdo con los enfoques antropológico, social y cultural, el envejecimiento "es una construcción sociocultural con significados diferentes según la época, las sociedades y las culturas; no solo tiene que ver con procesos biológicos, sino que es moldeada por normas y factores culturales y sociales" (2); por lo tanto, el significado de envejecimiento y vejez no es uniforme entre sociedades y culturas, sino que depende del contexto, del grupo poblacional y del tiempo en que se vive (2).

La familia, como grupo social, "provee la atención a sus integrantes tanto para la preservación de la salud como para su desarrollo" (3), con acciones de ayuda a la dignificación y funcionalidad del adulto mayor, a través de los vínculos familiares y comunitarios, es el grupo social donde se producen las primeras interacciones de los seres humanos y tiene múltiples responsabilidades y funciones con el bienestar y desarrollo de sus integrantes.

La perspectiva sistémica ofrece una mirada integradora para pensar a la familia de un adulto mayor, así como las funciones y roles que cada uno de sus miembros desempeñan, definiendo relaciones específicas y diferenciadas. Bateson et al. (4), fundamentados en los planteamientos de Bertalanffy (1950), pensaron en las familias como sistemas abiertos en interacción unos con otros, que constituyen una unidad como un conjunto 
de interacciones. La familia como un sistema permite observar la unidad familiar en términos de interacciones constantes entre todos sus integrantes y sus relaciones con otros grupos sociales externos al sistema familiar.

La familia como unidad de observación en la investigación permite identificar las relaciones, las interacciones y los sistemas que participan en el cuidado del adulto mayor. El hogar es el lugar ideal para interactuar con la familia, pues permite una observación más profunda de la dinámica familiar, es decir, la capacidad de la familia de cumplir las funciones propias (5) y necesarias para el anciano. Dado que en Villavicencio no se conoce la comprensión que tienen las familias acerca del adulto mayor y el proceso de envejecimiento de los individuos, fue necesario, desde enfermería, profundizar en aspectos de las familias y conocer su entorno familiar, de tal manera que el estudio responda a las necesidades del contexto y permita preparar a la comunidad académica para el cambio demográfico en las comunidades.

El objetivo de la investigación fue conocer el entorno familiar de los adultos mayores de los centros de vida (CV) del municipio de Villavicencio, con el propósito de construir, a partir de los resultados, elementos orientadores para la formación de cuidadores profesionales y el mejoramiento de la calidad de vida del adulto mayor.

\section{Método}

Investigación cualitativa (6) con enfoque etnográfico focalizado (7). Se buscó el punto de vista de los cuidadores de los $\mathrm{CV}$, así como el de los adultos mayores y sus familias. Se indagaron, describieron e interpretaron los hechos e interacciones, tal como ocurre en el contexto donde se encuentran los individuos (8); en este caso, la experiencia de envejecimiento y el entorno social y familiar donde habita el adulto mayor.

\section{Fase exploratoria}

Entre los $19 \mathrm{CV}$ de Villavicencio, se seleccionaron al azar tres: La Nohora, La Ceiba y La Cuncia. Una primera visita a cada CV permitió presentar el proyecto, obtener la autorización de las directivas y seleccionar a los participantes. Desde una concepción flexible del entorno familiar, se consideraron tres tipos de informantes clave: 1) los adultos mayores, alrededor de quienes iban a girar las observaciones y las entrevistas; 2) el cuidador del adulto mayor en el CV y 3 ) el principal cuidador en la familia.

\section{Fase de trabajo de campo}

Las primeras interacciones con los adultos mayores buscaron establecer un clima de mutua confianza y colaboración. El acercamiento a las familias se facilitó mediante la persona que llevaba al adulto mayor al CV. Se realizaron, en promedio, cuatro visitas a cada CV y cuatro visitas domiciliarias por cada familia. Durante cerca de 50 horas se observaron las interacciones en los CV y en los hogares; para esto, al terminar la jornada, se acompañó al adulto mayor en su regreso a casa. En el diario de campo se registró lo que los participantes creían, hacían, sabían y usaban como miembros activos del entorno inmediato del adulto mayor. Las entrevistas abiertas fueron conversaciones discontinuas que se grabaron. En cada visita se validaba lo observado y registrado hasta ese momento y se avanzaba tratando de profundizar en diferentes aspectos de la cotidianidad del adulto mayor. 


\section{Fase de análisis}

El análisis fue simultáneo con el trabajo de campo. El contenido de las observaciones y de las entrevistas fue convertido en unidades textuales. Los datos obtenidos se sometieron inicialmente a un proceso de codificación abierta, a fin de obtener una visión global acerca de lo observado y dicho. Se preestablecieron cuatro categorías: percepciones sobre el proceso de envejecimiento vivido u observado, mantenimiento de la familia, dinámicas familiares e interacciones alrededor de la identificación y satisfacción de las necesidades de cuidado del adulto mayor. Con los códigos que expresaban asuntos y sentidos similares se configuraron subcategorías, que posteriormente se contrastaron hasta identificar las categorías que expresaban componentes diferenciados del entorno familiar de los adultos mayores. Los hallazgos se describieron, interpretaron y contrastaron con la teoría. A manera de ejemplo, la referencia numérica utilizada en las narrativas fue: FM1 (familia), AM1 (adulto mayor), CU1 (cuidador CV) y la numeración corresponde al participante.

Se tuvieron en cuenta las consideraciones éticas planteadas en la Resolución 8430 de 1993 del Ministerio de Salud de Colombia (9): autorizaciones, aval bioético y consentimiento informado de los participantes para la observación, la entrevista, la grabación y la difusión de los resultados, lo que garantiza absoluta confidencialidad de la información y tratamiento anónimo del informante. La investigación siguió los principios internacionales de investigación establecidos en la Declaración de Helsinki de 1975 de la Asociación Médica Mundial, la Resolución 8430 de 1993 y contó con la aprobación del Comité de Bioética de la Universidad de los Llanos.

\section{Resultados y discusión}

Fueron tres adultos mayores (dos mujeres y un hombre) entre 65 y 85 años, independientes, con primaria incompleta, un viudo y dos divorciados, en el régimen de salud subsidiado y un promedio de hijos de 5,5. Los cuidadores familiares fueron dos hijas y un nieto, con un promedio de edad de 35 a 55 años, con primaria incompleta, bachillerato incompleto y universitario incompleto, régimen de salud dos subsidiados y uno contributivo. Los tres cuidadores entrevistados de los CV fueron mujeres, con una edad promedio de 45 años, con bachillerato incompleto. A continuación, se presentan las categorías que emergen en el análisis.

\section{Categoría I: entre aceptación o resignación de ser un adulto mayor}

El envejecimiento es percibido como la etapa final de la vida, asociada a cambios físicos, de actuar y la poca aceptación en el adulto mayor y la familia. Para D’Hyver y Gutiérrez (10), es un proceso de desarrollo humano que requiere adaptación a distintas características naturales de esta fase de la vida. Estos factores suponen una serie de desafíos y dificultades, entre los cuales puede mencionarse: físicos con una mayor vulnerabilidad a enfermar, cambios psicológicos y experiencias vitales de gran transcendencia como la sensación de cercanía de la muerte.

Cambios físicos y en la forma de ser "Físicamente se vuelve uno feo, todo le queda uno feo, todo arrugado, todo vuelto nada (risa), se pone malgeniado" (AM2). "Algunos cambian en la forma de ser" (CU2). "Él se volvió de malgenio, después de que se puso viejo" (FM2). "Le ha vuelto más mal genio" (FM3). "La vejez, llegan las enfermedades y que de pronto que como están enfermos se ponen de mal genio" (CU1). "A mí se me olvida todo" (AM1). "La edad poco a poco van perdiendo memoria, no ven bien" (CU2).

El estudio indica los cambios propios del envejecimiento, que para algunos son aceptados como parte del ser humano y en otros son rechazados. En investigaciones similares lo presentan como declives fisiológicos, 
a sentir un deterioro progresivo de los órganos; muchos adultos mayores consideran el envejecimiento como un proceso normal, como una posibilidad de hacer cosas nuevas, como un regalo divino para disfrutar y para poder compartir la experiencia y los aprendizajes con los más jóvenes e incluso con sus pares (1), manifestando su plenitud por esta etapa de la vida, también como un fenómeno presente a lo largo del ciclo vital desde el mismo proceso de la concepción hasta la muerte (11).

Le duele todo y no sirve para nada: "Cuando no le duele un brazo, un pie, una mano o la cabeza" (AM1). "El cuerpo le falla, tal como me pasa a mí que estoy enfermo" (AM3). "Yo al menos pues estoy enferma, pero me siento con salud" (AM1). "La vejez, que uno no sirve casi para nada, es tremenda" (FM1). "La vejez es una etapa dura, porque muchas veces no hay quien ayude" (FM2). "Dicen que no pueden hacer nada, no sirven para nada, es un estorbo para la familia" (CU2). "La verdad, yo le pido mucho a Dios que el día que ya no pueda moverme que no me deje sufrir” (AM2).

El estudio indica que el envejecimiento causa deterioro físico y discapacidad. Se observa poca aceptación frente a los procesos de envejecer, una etapa de pérdida y añoranzas, de cambios físicos y en la forma de ser, una inconformidad por las condiciones sociales y económicas en los adultos mayores. En coherencia con investigaciones donde la imagen del envejecimiento en la sociedad actual es negativa y es expresada como sinónimo de enfermedades, deterioro físico y mental (12), con una fuerte demanda de cuidado por parte de la familia $(13,14)$.

¡La vejez! Es una vaina que todo el mundo va para allá: "Que bonito llegar uno a viejito y uno entenderse, morir juntos y eso es bonito" (AM2). "Pues yo pienso de la vejez que es una vaina que en la que todo el mundo va para allá; lo único es tener capital para llegar a la vejez" (FM3). "Haber, la vejez es como el transcurso de la vida" (CU1). "La vejez es una etapa con experiencia, empiezan las arruguitas de juventud" (CU2). "Primero que todo son personas que le faltan cariño" (CU3).

El envejecimiento como etapa del ciclo vital. Esto también refleja la construcción cultural del envejecimiento como una pérdida en la vida social, esto es, se configura un imaginario colectivo que se manifiesta en las acciones y los recursos que tiene el adulto mayor en su entorno. El envejecimiento activo que promueve la OMS tiene el propósito de que los adultos mayores disfruten una vida saludable con autonomía e independencia que conlleve calidad de vida. En este sentido, las personas, a medida que envejecen, tienden al sedentarismo, porque disminuyen sus capacidades, aumentan sus limitaciones, reducen sus actividades, se vuelven dependientes y se afecta su calidad de vida (15).

\section{Categoría II: los menesteres de la vejez}

La categoría revela las necesidades del adulto mayor: condiciones sociales, viviendas con deficiencia de servicios públicos y ambientes inseguros tanto en la vivienda como en el contexto comunitario que pueden afectar la integridad del adulto mayor. "Existen muchos fenómenos que influyen en la manera en la que la persona mayor se comporta de cara a la gente que lo rodea; la declinación de las funciones fisiológicas y habilidades cognitivas, el nivel de apoyo familiar, la soledad y el abandono, la jubilación siendo una las mayores fuentes de estrés". De ahí que la familia se constituya en el entramado social más importante (16) en la satisfacción de sus necesidades.

No se puede trabajar: "Yo hace un año que estoy enfermo sin poder hacer nada, sin poder ganarme un peso" (AM3). "No, no de verdad que el apoyo que la familia le dan es muy poco, y muchos trabajan en las cositas que le salgan" (CU3). "Él no pueda hacer gran cosa de trabajo" (FM1).

Las necesidades del adulto mayor se manifiestan en sentirse útiles, en el bajo nivel socioeconómico sumado a otros factores de ingreso que hacen que el cuidado sea deficiente y de baja calidad. La Comisión Económica para América Latina, en 2014, estableció prioridades para el adulto mayor, como es la seguridad económica, sistemas de proyección social, participación en la vejez que incluya la participación en la concertación 
y control de políticas y programas, participación comunitaria, voluntariado y redes de parentesco, que contribuya a disminuir la desigualdad extrema en esta población (17), reflejadas en las narrativas de los actores sociales de la investigación.

Cualquier cosa es cariño: "Los hijos me ayudan con algo" (AM3). "Los problemas son económicos, porque a ratos no hay plata" (FM2). "Si tienen modos económicos, me colaboran con cualquier peso" (AM2). "Pues el viene y por acá me da un vistazo y se va, pero algo es algo peor es nada, cualquier cosa es cariño" (AM2). "No hay ningún familiar que nos ayude" (FM1). "Ellos sí tienen la familia, a veces los tratan bien y otras veces no" (CU2). "Vienen muy pocas veces y le dan una miradita al viejito" (CU1).

El estudio refleja la permanente inconformidad del adulto mayor por la carencia de afecto y de recursos para satisfacer sus necesidades básicas, como son el alimento, la vivienda y la salud. Los cuidadores responsabilizan a las familias por el abandono a que son sometidos los adultos mayores. Estudios indican que la calidad de vida (18) y la capacidad funcional (19) de los adultos mayores dependen en gran medida de la seguridad económica y del apoyo de la familia.

La compañia, el cuidado y la ayuda para los viejitos: "Ella me mira mi ropita, me cuida adonde voy, al médico" (AM1). "Vive en la casa con el esposo" (CU3). "Lo cuidamos cuando él está enfermo; él es una persona que no se deja llevar" (FM2). "Yo mismita me cuido. ¿Quién me va cuidar? Vienen y me visitan para el día de la madre" (AM2). "Generalmente, aquí en la casa estamos pendientes de ella" (FM3). "Todos cuidan al viejito, con la comidita, la camita, es un anciano ¡figúrese!” (FM1). "Mi esposa es la que me ayuda, para el aseo de la ropita, cuando tengo necesidad de la comida, y sed" (AM3). "La familia están pendientes de los controles médicos, de que venga al Centro Vida" (CU1).

El apoyo social y familiar es otra necesidad evidente en el adulto mayor. Las narrativas indican las necesidades de acompañamiento y el abandono social que inicia por la muerte de la pareja, de los amigos, la pérdida de su vida laboral y el control económico. Estudios muestran una situación generadora de sentimiento de soledad, que hace que los ancianos se sientan desprotegidos y vulnerables (19). A pesar del sentimiento general de satisfacción con respecto al apoyo familiar, algunos de ellos expresaron sentirse maltratados emocionalmente y socialmente. Investigaciones indican que los trastornos afectivos (20) pueden verse exacerbados por la falta de apoyo familiar y por la disminución de las interacciones sociales, lo que puede conducir a un aislamiento significativo que altera la calidad de vida de esta población.

\section{Categoría III: las relaciones de la familia cuando se tiene a un adulto mayor}

Se observan tres dinámicas de relaciones familiares: una, son los conflictos entre los integrantes de la familia y el adulto mayor. La otra, en menor proporción, son las expresiones de afecto, respeto y solidaridad de los integrantes de la familia y los cuidadores de los CV. La última está relacionada con el distanciamiento y desconocimiento de la familia con la institución donde pasa el mayor tiempo del día el adulto mayor. "Las concepciones de valía de un adulto mayor varían de un país a otro. En algunos sitios, los adultos mayores son reverenciados por su conocimiento; en otros se han vuelto casi invisibles, ignorados por la población en general y en muchos casos por sus familias" (21).

Discusiones y peleas jeso si no faltan!: "Aunque sí, a ratos, se pone como cansón” (FM1). "La discusiones y peleas con mi esposo, pues eso sí no faltan, son más bien mi nieta" (AM2). "Pero yo no me dejo de ellos ni por el carajo; ellos piensan en regañarme y yo no me les dejo" (AM1). "Pues problemas con los hijos es por lo desordenados, por borrachos" (AM3). "El cuidado es regular porque nosotros a veces queremos ayudarlo a él, pero no se deja, es muy conflictivo, nada le gusta” (FM2).

El estudio reporta lo que puede generar el cuidado de un adulto mayor en las relaciones intrafamiliares, muchas veces por desconocimiento en el manejo de las situaciones de salud y la integración social e incomodidad por los cambios emocionales del adulto mayor. Otro factor importante en el plano comunitario 
es el destierro por la guerra, que genera en los adultos mayores sentimiento de desarraigo, tristeza y abandono, donde dos de ellos fueron desplazados de su territorio. Los entornos sociales favorables del adulto mayor están dados por el trato que se le da a esta población; investigaciones reportan maltrato (1) familiar, por las condiciones de discapacidad y su dependencia económica. La familia cumple un papel importante en la generación de espacios adecuados para suplir las necesidades físicas y mentales del adulto mayor, si se tiene en cuenta que la capacidad funcional disminuye notablemente (22), y que la variable de enfermedad está presente y asociada al simple hecho de estar viejo (23).

Agradecidos por la ayuda recibida: "Ellos viven muy agradecidos con el CV, hacen oración, me motiva a mí a seguir trabajando ayudándolos en lo que más se puede" (CU1). "Yo vivo muy contento con la ayuda que me da este lugar $(\mathrm{CV})$ ¡Sí!, como dice la palabra de Dios guardar lo bueno y desechar lo malo, con las muchachas que nos atienden" (AM3).

Los adultos mayores son conscientes de su estado de dependencia y sentimiento de agradecimiento por la poca o mucha ayuda que reciben de las instituciones, consideran que son espacios que les permiten evadir en alguna medida las actividades asignadas por sus familias como el cuidar de los nietos y bisnietos, asumir responsabilidades económicas, y otras que consideran tareas cumplidas en el ciclo de la vida. Investigaciones indican que la familia y la comunidad son fuentes importantes de apoyo durante la vejez; de ello depende mucho la calidad de vida y el estado de salud mental de los ancianos, esencial para interactuar y participar en las actividades propias de la familia, la comunidad y la misma sociedad (20). Ello aumenta la autoestima y mejora las relaciones con el entorno.

Ninguno de nosotros ha ido: "Como familia no participo en las actividades del CV, ninguno de nosotros ha ido" (FM1). "Yo no conozco el CV. A mí me parece chévere que vaya allá, porque mantiene ocupado, le dan de comer, lo sacan a pasear y él se distrae mucho; en cambio de estar aquí, jodiendo la vida" (FM2). "La familia poco le gusta venir al CV, no me acompañan" (AM3). "No conozco la familia, ninguna profesional ha ido a la casa" (LD2). "Para ellos el hogar es acá, el Centro Vida, tenemos muchos que viven solos" (LD1).

$\mathrm{El}$ abandono del adulto mayor por parte de su familia es muy evidente en el estudio. Los CV hacen muy poco esfuerzo para integrar a la familia en los programas que desarrolla, lo que va en contravía con el propósito de la Ley 1276 de 2009 (24), donde los CV están orientados a brindar una atención integral, garantizar y satisfacer necesidades como alimentación, salud, interacción social, deporte, cultura y recreación, actividades productivas y espirituales.

\section{Conclusiones}

La no aceptación de los cambios físicos y psicológicos en el adulto mayor — por ejemplo, en su familia- y la falta de tolerancia de su entorno se refleja permanente en las narrativas, pues ello los induce a la soledad $\mathrm{o}$ al aislamiento familiar, visibles en las observaciones durante el estudio. El verdadero rol que desempeña el adulto mayor en una sociedad no se vislumbra en el contexto estudiado. Sería importante plantear algunos propósitos y acciones que mejoren sustancialmente el manejo que se le da al adulto mayor, en los ámbito familiar, comunitario e institucional en la región.

Dentro de las políticas públicas se debe orientar a la familia y sociedad para ser un adulto mayor del mañana, y destacar que la vejez está relacionada con la manera en que se han vivido los anteriores ciclos de vida y las relaciones intergeneracionales que se hayan producido. Así mismo, generar actividades de integración para fortalecer las relaciones del adulto mayor con su entorno familiar, comunitario e institucional, y seguir en el proceso de formación de profesionales y cuidadores, para comprender las dinámicas del envejecimiento y fortalecer las acciones en el cuidado del adulto mayor. 


\section{Limitaciones}

La accesibilidad en algunos CV y en oportunidad, al no encontrar el cuidador familiar.

\section{Agradecimientos}

Las autoras expresan los agradecimientos a las personas adultas mayores, sus familias y los líderes de los centros vida. A la Secretaría Social y de Participación Comunitaria de la ciudad de Villavicencio. A la Universidad de los Llanos, por el aval y el apoyo económico. A las estudiantes principiantes de investigación del Programa de Enfermería, Facultad de Ciencias de la Salud. Por último, al grupo de investigación GESI.

\section{Referencias}

1. Flórez CE, Villar L, Puerta N, Berrocal LF. El proceso de envejecimiento de la población en Colombia: 1985-2050. Bogotá: Editorial Fundación Saldarriaga Concha; 2015.

2. Dulcey-Ruiz E. Envejecimiento y vejez: categorías y conceptos. Bogotá: Fundación Cepsiger y Red Latinoamericana de Gerontología; 2015.

3. Ostiguín-Meléndez RM, Bustamante-Edqén S. Dimensiones del cuidado en familias urbanas mexicanas. Enferm Universitaria. 2016;13(2):80-9. https://www.dx.doi.org/10.1016/j.reu.2016.03.007.

4. Bateson G, et al. Interacción familiar. Buenos Aires: Tiempo Contemporáneo; 1971.

5. Jiménez-Aguilera B, Baillet-Esquivel LE, Ávalos-Pérez F, Campos-Aragón L. Dependencia funcional y percepción de apoyo familiar en el adulto mayor. Atención Familiar. 2016;23(4):129-33. https://www.dx.doi.org/10.1016 /j.af.2016.08.002.

6. Taylor J, Bogdan R. Introducción a los métodos cualitativos de investigación. Barcelona: Paidós; 1984.

7. Mauss M. Manual de etnografía. Buenos Aires: Fondo de Cultura Económica; 2006.

8. Boyle J. Estilos de etnografía. En: Morse J. Asuntos críticos en los métodos de investigación cualitativa. Medellín: Universidad de Antioquia; 2003.

9. Resolución 8430 del 4 de octubre 1993, por la cual se establecen las normas científicas, técnicas y administrativas para la investigación en salud [internet]. Disponible en: https://www.minsalud.gov.co/sites/rid/Lists/Bibliotec aDigital/RIDE/DE/DIJ/RESOLUCION-8430-DE-1993.PDF.

10. D’Hyver C, Gutiérrez Robledo LM. Geriatría. 3a ed. México: Manual Moderno; 2014.

11. Alvarado García AM, Salazar Maya ÁM. Análisis del concepto de envejecimiento. Gerokomos [internet]. 2014 Jun [citado 2017 ago 8];25(2):57-62. https://www.dx.doi.org/10.4321/S1134-928X2014000200002.

12. Benavides-Caro CA. Deterioro cognitivo en el adulto mayor. Rev Mex Anestesiol [internet]. 2017;40(2):107-12. https://www.dx.doi.org/10.3389/fnagi2016.00253.

13. Cardona AS, Duque MG, Arango DC, Cardona AS. Risk of cognitive impairment in elderly people in the subregions of Antioquia, Colombia. Rev Bras Estud Populacao. 2016;33(3):613-28. http:// dx.doi.org/10.20947/S0102-30982016c0008.

14. Guevara E. The importance of knowing "than expected for age" in cognitive assessment of the elderly patient. Rev Med Chile. 2016;144(12):16-21. https://www.dx.doi.org/10.4067/S0034-98872016001200016.

15. Aguilar Hernández RM. El modelo de enfermería Roper-Logan-Tierney en el adulto mayor. México: McGraw Hill; 2012.

16. Quiroga Capovilla H. Curso práctico de auxiliar de geriatría: Asistencia domiciliaria a personas de la tercera edad. Manual de formación profesional. Málaga, España: Daly; 2014.

17. Comisión Económica para América Latina y el Caribe (Cepal). Panorama social de América Latina [internet]. 2014. Disponible en: https://www.repositorio.cepal.org/handle/11362/37626. 
18. Varela Pinedo LF. Health and quality of life in the elderly. Rev Peru Med Exp Salud Pública. 2016;33(2):199-201. https://www.dx.doi.org/10.17843/rpmesp.2016.332.2196.

19. Zapata-López BI, Delgado-Villamizar NL, Cardona-Arango D. Social and family support to the elderly in urban areas. Rev Salud Pública. 2015;17(6). https://www.dx.doi.org/10.15446/rsap.v17n6.34739.

20. Tello-Rodríguez T, Alarcón RD, Vizcarra-Escobar D. Mental health in older adults: Major neurocognitive, affective, and sleep disorders. Rev Peru Med Exp Salud Pública. 2016;33(2):342-50. https://www.dx.doi.org/1 0.17843/rpmesp.2016.332.2211.

21. Eckman M. Enfermería geriátrica. México: Manual Moderno; 2012.

22. Cortés-Muñoz C, Cardona-Arango D, Segura-Cardona Á, Garzón-Duque MO. Demographic, social, physical and mental factors associated with functional capacity in the elderly. Antioquia, Colombia. Rev Salud Pública. 2016;18(2):167-78. https://www.dx.doi.org/10.15446/rsap.v18n2.49237.

23. Melguizo Herrera E, Bertel de la Hoz A, Paternina Osorio D, Felfle Fuentes Y, Porto Osorio L. Cognitive stimulation of elderly residents in social protection centers in Cartagena, 2014. Rev Colomb Psiquiatr. 2016;46(4):229-36. https://www.dx.doi.org/10.1016/j.rcp.2016.09.008.

24. Ley 1276 del 5 de enero de 2009, a través de la cual se modifica la Ley 687 del 15 de agosto de 2001 y se establecen nuevos criterios de atención integral del adulto mayor en los centros de vida [internet]. Diario Oficial n. ${ }^{\circ} 47.223 \mathrm{del} 5$ de enero de 2009. Disponible en: http://www.secretariasenado.gov.co/senado/basedoc/ ley_1276_2009.html

\section{Notas}

* Artículo original de investigación

Conflictos de interés: los autores declaran no tener conflictos de interés.

Financiación: la investigación fue autorizada y financiada por la Universidad de los Llanos, Villavicencio,

Colombia.

\section{Licencia Creative Commons CC BY 4.0}

Cómo citar: Salamanca-Ramos E, Velasco Páez ZJ, Díaz Viatela CD. Entorno familiar del adulto mayor de los centros vida de la ciudad de Villavicencio, Colombia. Investig Enferm Imagen Desarr. 2019;21(1). https://www.doi.org/10.11144/Javeriana.ie21-1.efam 